\title{
Small-angle neutron scattering study of structure and interaction during salt-induced liquid-liquid phase transition in protein solutions
}

\author{
A. J. Chinchalikar, ${ }^{1}$ V. K. Aswal, ${ }^{1}$ J. Kohlbrecher, ${ }^{2}$ and A. G. Wagh ${ }^{1}$ \\ ${ }^{1}$ Solid State Physics Division, Bhabha Atomic Research Centre, Mumbai-400085, India \\ ${ }^{2}$ Laboratory for Neutron Scattering, Paul Scherrer Institut, CH-5232 PSI Villigen, Switzerland
}

(Received 23 April 2013; published 18 June 2013)

\begin{abstract}
The liquid-liquid phase transition (LLPT) in aqueous salt solutions of lysozyme protein has been studied by small-angle neutron scattering. Measurements have been carried out on fixed protein concentration with varying salt concentration approaching LLPT. The data are fitted considering protein interaction by the two Yukawa (2Y) potential which combines short-range attraction and long-range repulsion. We show that LLPT arises because of enhancement of non-DLVO (Derjaguin-Landau-Verwey-Overbeek) short-range attraction without any conformational structural change of the protein. The salt concentration required for LLPT as well as corresponding short-range attraction decreases significantly with increase in protein concentration.
\end{abstract}

DOI: 10.1103/PhysRevE.87.062708

PACS number(s): 87.15.km, 87.15.Zg, 82.70.Dd, 61.05.fg

\section{INTRODUCTION}

Proteins are macromolecules made of amino acids, which perform structural, transport, catalytic, sensory, and many other important biological functions [1,2]. In solutions, they are known to exhibit rich phase behavior [3,4]. The variation of different solution conditions such as concentration, temperature, $p \mathrm{H}$, and ionic strength can induce the protein solutions to undergo various phase transitions following the structural changes which are often preceded by the appropriate changes in interactions among the protein molecules [5,6]. It is believed that many of these phases of protein solutions can be explained by the interaction combining short-range attraction along with the long-range repulsion [7-10]. The tuning of these two interaction components can lead to different phase transitions including liquid-liquid phase transition (LLPT) [11]. In LLPT, the protein solution gets divided into two phases; one is rich in protein while the other is protein deficient [12]. The liquid-liquid phase is one of the important phases that can enhance a solid phase such as crystallization [13-15]. The recent simulations and theory predicts that the LLPT reduces the nucleation barrier thereby elevating crystal nucleation [16]. The LLPT is metastable with respect to other phase transitions. Thus, this phase transition is interesting not only because it competes with formation of other phases but also it can provide a distinctive kinetic route for these other processes. The LLPT is also important from the medicinal point of view related to cold cataracts observed in eye lenses of certain mammalian species [17].

There have been a number of studies to understand different aspects of LLPT in protein solutions [18-23]. Most of the studies on LLPT were carried out in the presence of salts, which is believed to influence structure as well as interaction in protein solution leading to LLPT. The different structural forms of protein molecules, from monomers to their clusters, have been reported in their salt solutions [18,21-23]. These different forms of protein structures strongly depend on the protein and salt used. However, no direct correlation between these structures and LLPT has been found. The interaction of proteins in the absence of salt or at low ionic strengths can be successfully modeled by the Derjaguin-Landau-VerweyOverbeek (DLVO) potential which combines short-range van der Waals attraction with long-range double layer repulsion [24]. At higher salt concentrations and on approaching LLPT, the DLVO potential does not work because of emergence of non-DLVO contributions [25-28]. This non-DLVO potential has been mostly modeled by the Baxter's sticky hard sphere potential interacting through a range much smaller than the size of the protein. The depth of the potential rises with the increase in salt concentration and LLPT is believed to occur when it becomes significantly larger than the thermal energy of the particle [29]. The potential is however oversimplified due to its (i) confinement over a very narrow region and (ii) distance $(r)$ independence within that region [30,31], which limits the applicability of this potential. Recently, the two Yukawa (2Y) potential has been used to take account of both the DLVO and non-DLVO interactions in protein solutions [32]. The strength and range of both attractive and repulsive interactions for this potential can be determined. In this paper, we have examined the evolution of structure and interaction in protein salt solution undergoing LLPT by small-angle neutron scattering (SANS).

We have performed SANS measurements on aqueous lysozyme solutions over a wide range of protein concentration (1-20 wt \%) with varying concentration of $\mathrm{NaCl}$ approaching LLPT. Lysozyme (M.W. 14.4 kDa) has been used as a model protein which is one of the most commonly studied in the literature because of its structural robustness [33]. SANS is an ideal technique to probe both structure and interaction in such protein systems $[34,35]$. The measured scattering intensity in SANS is the product of the form factor and the structure factor, where the form factor provides information on the structure (shape and size) of particles and the structure factor depends on the interaction between particles. The SANS data are analyzed using the $2 \mathrm{Y}$ potential for protein molecules interacting via short-range attraction and long-range repulsion.

\section{EXPERIMENT}

Hen egg white lysozyme (three times crystallized and lyophilized) was obtained from Fluka and used as obtained. All the samples were prepared in $20 \mathrm{~m} M$ phosphate buffer of $p \mathrm{D} 7.0$ in $\mathrm{D}_{2} \mathrm{O}\left(99.9\right.$ at. \%) as solvent (instead of $\mathrm{H}_{2} \mathrm{O}$ ) for getting better contrast in SANS experiments. Small-angle 
neutron scattering measurements were carried out at the SANS-I facility, Swiss Spallation Neutron Source SINQ, Paul Scherrer Institut, Switzerland [36]. The wavelength of the neutron beam used was $6 \AA$. The experiments were performed at sample-to-detector distances of 2 and $6 \mathrm{~m}$ to cover wave vector transfer $Q(=4 \pi \sin \theta / \lambda$, where $2 \theta$ is the scattering angle and $\lambda$ is the wavelength of incident neutron) in the range of $0.01-0.3 \AA^{-1}$. The scattered neutrons were detected using a two-dimensional $96 \mathrm{~cm} \times 96 \mathrm{~cm}$ detector. The measurements were performed on different protein concentrations $(1,2,5$, 10 , and $20 \mathrm{wt} \%$ ) of lysozyme solutions with varying $\mathrm{NaCl}$ concentrations approaching LLPT. The samples with different protein and salt concentrations were obtained by diluting the concentrated stock solutions of protein (25 wt \%) and salt (4 $M$ ). The stock solutions were prepared by dissolving the known amounts of protein and salt in buffer solution and kept overnight to get the protein fully dissolved. The measurements were carried out on the freshly prepared samples. All the data were corrected and normalized to absolute scale using BERSANS-PC data processing software [37].

\section{SMALL-ANGLE NEUTRON SCATTERING ANALYSIS}

SANS is an elastic scattering technique in which one measures coherent differential scattering cross section per unit volume $(d \Sigma / d \Omega)$ as a function of $Q$. For a monodispersed system of protein macromolecules, $d \Sigma / d \Omega$ can be expressed as [34]

$$
\frac{d \Sigma}{d \Omega}(Q)=n\left(\rho_{p}-\rho_{s}\right)^{2} V_{p}^{2} P(Q) S(Q)+B,
$$

where $n$ is the number density of macromolecules; $\rho_{p}$ and $\rho_{s}$ are scattering length densities of macromolecule and solvent, respectively. $V_{p}$ is the volume of the macromolecule. $P(Q)$ is the square of the form factor $F(Q)$ which contains information about the shape and size of macromolecule. $S(Q)$ represents the interparticle structure factor and is determined by the interaction between macromolecules. $B$ is a constant term due to incoherent background which is mainly from the hydrogen content in the sample.

$P(Q)$ is calculated for the prolate ellipsoidal shape of the protein macromolecule and is given by

$$
\begin{gathered}
P(Q)=\int_{0}^{1} F^{2}(Q, \mu) d \mu, \\
F(Q, \mu)=\frac{3(\sin x-x \cos x)}{x^{3}}, \\
x=Q\left[a^{2} \mu^{2}+b^{2}\left(1-\mu^{2}\right)\right]^{1 / 2},
\end{gathered}
$$

where $a$ and $b=c$ are semimajor and semiminor axes of the protein macromolecule. $V_{c}\left(=4 \pi a b^{2} / 3\right)$ is the total volume of the macromolecule. The variable $\mu$ is the cosine of the angle between the directions of $a$ and $Q$.

In the case of an isotropic system, $S(Q)$ can be written as

$$
S(Q)=1+4 \pi n \int[g(r)-1] \frac{\sin Q r}{Q r} r^{2} d r,
$$

where $g(r)$ is the radial distribution function. It is the probability of finding the particle at a distance $r$ from a reference particle centered at the origin. The $g(r)$ is governed by the form of the potential $V(r)$. It has been calculated using the two Yukawa potential under mean spherical approximation [32]. The two Yukawa potential having four parameters $\left(K_{1}, K_{2}, Z_{1}\right.$, and $\left.Z_{2}\right)$ is expressed by

$$
\frac{V(r)}{k_{B} T}=\infty(r \leqslant \sigma)=V_{2 Y} / k_{B} T(r>\sigma),
$$

where

$$
\begin{aligned}
\frac{V_{2 Y}(r)}{k_{B} T}= & -K_{1} \frac{\exp \left[-Z_{1}(r / \sigma-1)\right]}{r / \sigma} \\
& +K_{2} \frac{\exp \left[-Z_{2}(r / \sigma-1)\right]}{r / \sigma}
\end{aligned}
$$

and $\sigma\left[=2\left(a b^{2}\right)^{1 / 3}\right]$ is an effective hard sphere diameter of the protein macromolecule.

The second virial coefficient $\left(B_{2}\right)$ depends on the pair interaction between the macromolecules and can be calculated from the interaction potential as given by [38]

$$
B_{2}=\frac{2 \pi N_{A}}{M^{2}} \int\left[1-\exp \left(-V_{2 Y}(r) / k_{B} T\right)\right] r^{2} d r,
$$

where $N_{A}$ is Avogadro's number and $M$ is the molecular weight of the protein.

The analysis has been carried out by fitting experimental SANS data using Eq. (1). There are only four fitting parameters ( $K_{1}, K_{2}, Z_{1}$, and $Z_{2}$ ) describing the total interaction potential considered in the analysis of SANS data. The structural parameters of the protein are obtained from the dilute solution. The volume fraction of protein is kept constant as calculated from the concentration of the protein solution. Throughout the data analysis, corrections were made for instrumental smearing. The scattering profiles as given by Eq. (1) were smeared by the appropriate resolution function of the instrument to compare with the measured data. The parameters in the analysis were optimized by means of a nonlinear least-squares fitting program [39].

\section{RESULTS AND DISCUSSION}

Figure 1 is the room temperature $\left(30^{\circ} \mathrm{C}\right)$ phase diagram of a lysozyme protein- $\mathrm{NaCl}$ salt system as a function of protein

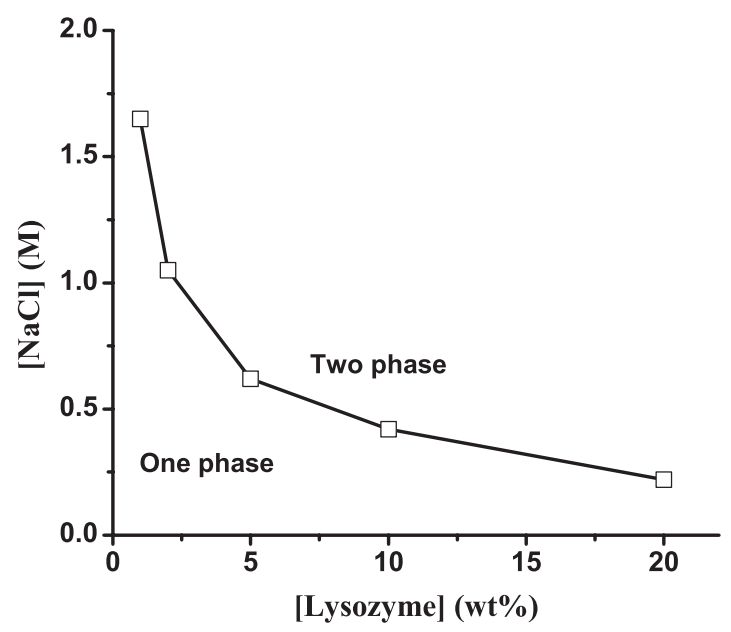

FIG. 1. The variation in LLPT salt concentration as a function of lysozyme protein concentration. 
concentration undergoing LLPT. The phase diagram provides $\mathrm{NaCl}$ concentration required for achieving LLPT where the protein solution undergoes phase transition from a one phase to a two phase system. The LLPT point is determined by the study of light transmission. It is also observed visually that the system becomes turbid at this point. The turbidity is the characteristic of LLPT arising from the protein-rich phase. It is observed that one phase region is significantly reduced with increasing protein concentration. These results are consistent with the fact that the solubility of protein is known to decrease with increasing protein concentration and therefore LLPT is observed at lower salt concentrations for higher protein concentrations [40]. The solubility and LLPT are basically connected as both are related with the hydration of the protein. The LLPT is believed to arise from enhanced hydrophobic attraction among the protein molecules due to entropy driven dehydration induced by the hydrated salt ions at the protein surface. This statement is based on some of our earlier work on salt-induced micellization of amphiphilic block copolymer [41] and clouding in surfactant micelles [42]. It had been found that the effect of increasing salt concentration is similar to that of increasing temperature. This salting out effect has been found to be more pronounced for the salts ions (both coions and counterions) when their hydrated size is large.

Figure 2 shows SANS data of 5 wt $\%$ aqueous lysozyme solution with varying concentration of $\mathrm{NaCl}$ approaching LLPT. The measured LLPT salt concentration for $5 \mathrm{wt} \%$ lysozyme solution is $0.62 \mathrm{M}$. It is observed that the scattering data change significantly in the lower $Q$ range $\left(Q<0.08 \AA^{-1}\right)$ whereas the data in the higher $Q$ range almost overlap each other with the increase in salt concentration. A correlation peak, an indication of repulsive interaction between charged protein molecules is seen for pure protein (no salt) solution. The correlation peak broadens along with the strong buildup of scattering intensity in the low $Q$ region. This buildup of scattering intensity has been believed to arise because of the evolution of attractive interaction among the protein molecules

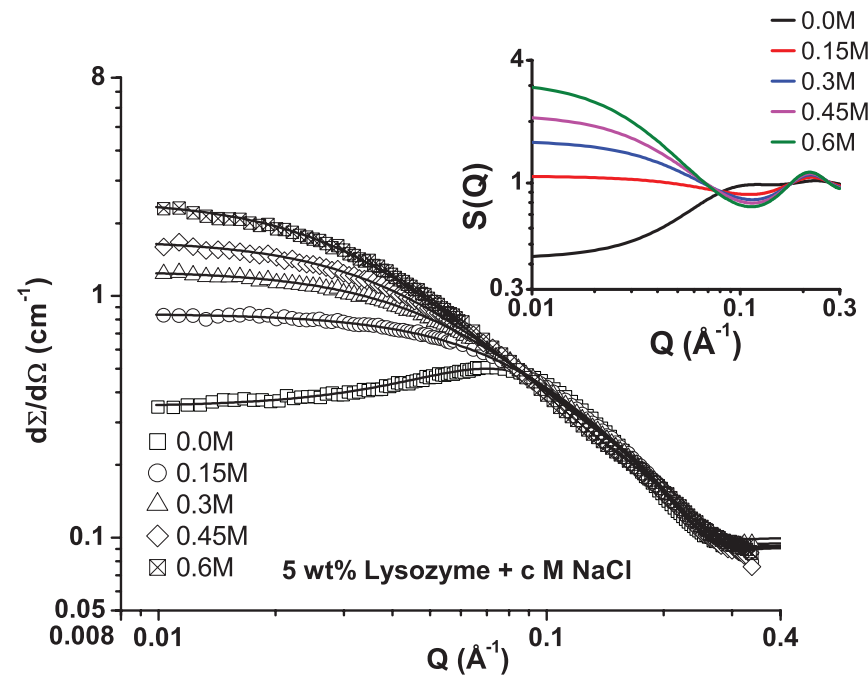

FIG. 2. (Color online) SANS data of $5 \mathrm{wt} \%$ lysozyme with varying $\mathrm{NaCl}$ concentration. The inset shows structure factor curves for the fitted SANS data. The curves in the inset from bottom to top in the low $Q$ region correspond to increasing salt concentration. in the presence of salt. The structure factor contribution in the scattering intensity at low $Q$ values is known to depend on the isothermal compressibility of the system and therefore diverges for attractive interaction as found in the present data. The data have been fitted with Eq. (1) in which $S(Q)$ is calculated for the two Yukawa potential [Eq. (6)] where the second Yukawa term is for the long-range repulsion between charged protein macromolecules and the first Yukawa term takes account of the short-range attraction induced by the presence of salt ions in protein solutions. The solid lines in Fig. 2 are the fitted curves of the experimental data. These data have been fitted with the form factor of the prolate ellipsoidal shape of the protein macromolecule [Eq. (2)]. The calculated values of semimajor and semiminor axes of lysozyme macromolecule are obtained from the dilute protein solution $(0.5 \mathrm{wt} \%)$ having values of 24 and $13 \AA$, respectively, and are found consistent with the literature $[43,44]$. The calculated structure factors using $2 \mathrm{Y}$ potential to fit the data are shown in the inset of Fig. 2. It is clear that the system is becoming more and more attractive on approaching LLPT with increasing salt concentration. The variations of attractive, repulsive, and total potential with varying salt concentration are shown in Fig. 3. It may be noted that short-range attraction
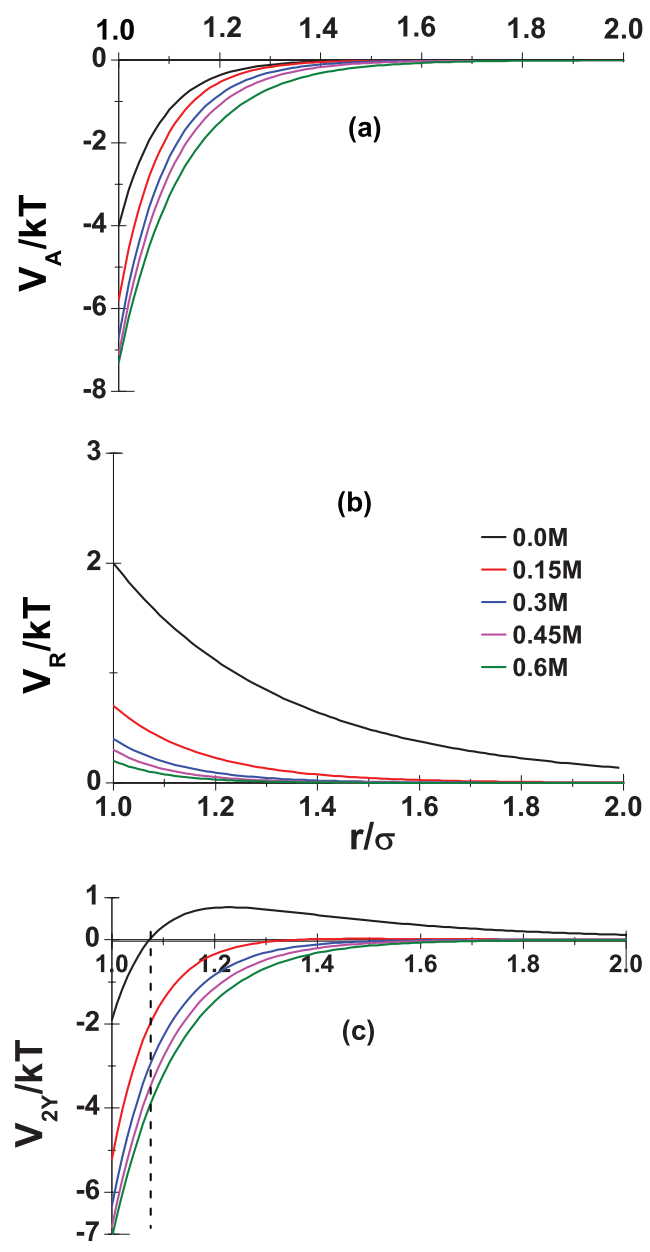

FIG. 3. (Color online) The calculated (a) attractive, (b) repulsive, and (c) total potential for $5 \mathrm{wt} \%$ lysozyme protein solution approaching LLPT. The curves from top to bottom in each case correspond to increasing salt concentration. 
TABLE I. The fitting parameters $\left(K_{1}, Z_{1}, K_{2}\right.$, and $\left.Z_{2}\right)$ of $2 Y$ potential for SANS data of 5 wt $\%$ lysozyme solution. The value of total potential at a distance that protein molecules experience through a hydration layer between them is also given. The calculated values of charge (in electron units) and ionic strength (I. S.) (in molar units) as obtained from $K_{2}$ and $Z_{2}$ are given in parentheses, respectively. The second virial coefficient $\left(B_{2}\right)$ is calculated from the fitted parameters of the potentials.

\begin{tabular}{|c|c|c|c|c|c|c|}
\hline $\begin{array}{l}\mathrm{NaCl} \\
\text { concentration } \\
(M)\end{array}$ & $K_{1}$ & $Z_{1}$ & $K_{2}$ (charge) & $Z_{2}$ (I. S.) & $V_{2 \mathrm{Y}} / k T$ & $\begin{array}{c}B_{2} \\
\left(\mathrm{~mol} \mathrm{ml} / \mathrm{g}^{2}\right)\end{array}$ \\
\hline 0.0 & 4.0 & 11.0 & $2.0(6.18)$ & $2.0(0.03)$ & - & $5.9 \times 10^{-4}$ \\
\hline 0.15 & 5.8 & 11.0 & $0.7(6.08)$ & $4.7(0.17)$ & -1.8 & $-2.1 \times 10^{-4}$ \\
\hline 0.3 & 6.7 & 9.5 & $0.4(5.86)$ & $6.4(0.32)$ & -2.6 & $-3.8 \times 10^{-4}$ \\
\hline 0.45 & 7.1 & 8.5 & $0.3(5.70)$ & $7.8(0.47)$ & -3.2 & $-4.8 \times 10^{-4}$ \\
\hline 0.6 & 7.3 & 7.0 & $0.2(5.10)$ & $8.5(0.56)$ & -3.5 & $-6.3 \times 10^{-4}$ \\
\hline
\end{tabular}

in salt-free protein solution is found to be similar to that as obtained from the van der Waals component of the DLVO potential. The increase in attractive interaction with salt can no longer be explained by the DLVO potential and is believed to be from non-DLVO components arising from dehydration properties of salt $[42,45]$. The fitted parameters of structure and interaction for the data (Fig. 2) are given in Table I. The globular structure of lysozyme protein remains unaltered with salt concentration and the changes observed in data are fitted using changes in the interaction potential modeled by the $2 \mathrm{Y}$ potential comprised of four fitting parameters $\left(K_{1}, K_{2}, Z_{1}\right.$, $Z_{2}$ ). Here, $K_{1}$ and $Z_{1}$ are the fitting parameters of the attractive potential to give the strength (proportional to $K_{1}$ ) and range (proportional to $1 / Z_{1}$ ), respectively. On the other hand, $K_{2}$ and $Z_{2}$ are the fitting parameters of the repulsive potential which provide the strength (related to effective charge) and range (related to ionic strength), respectively [46].

The values of effective charge and ionic strength as calculated from the $K_{2}$ and $Z_{2}$ are given in Table I; they have been found to follow the trend as expected with the increase in salt concentration. This suggests strength and range of repulsive potential decrease on addition of salt. The changes in the repulsive potential parameters (effective charge and ionic strength) of the $2 \mathrm{Y}$ potential on approaching
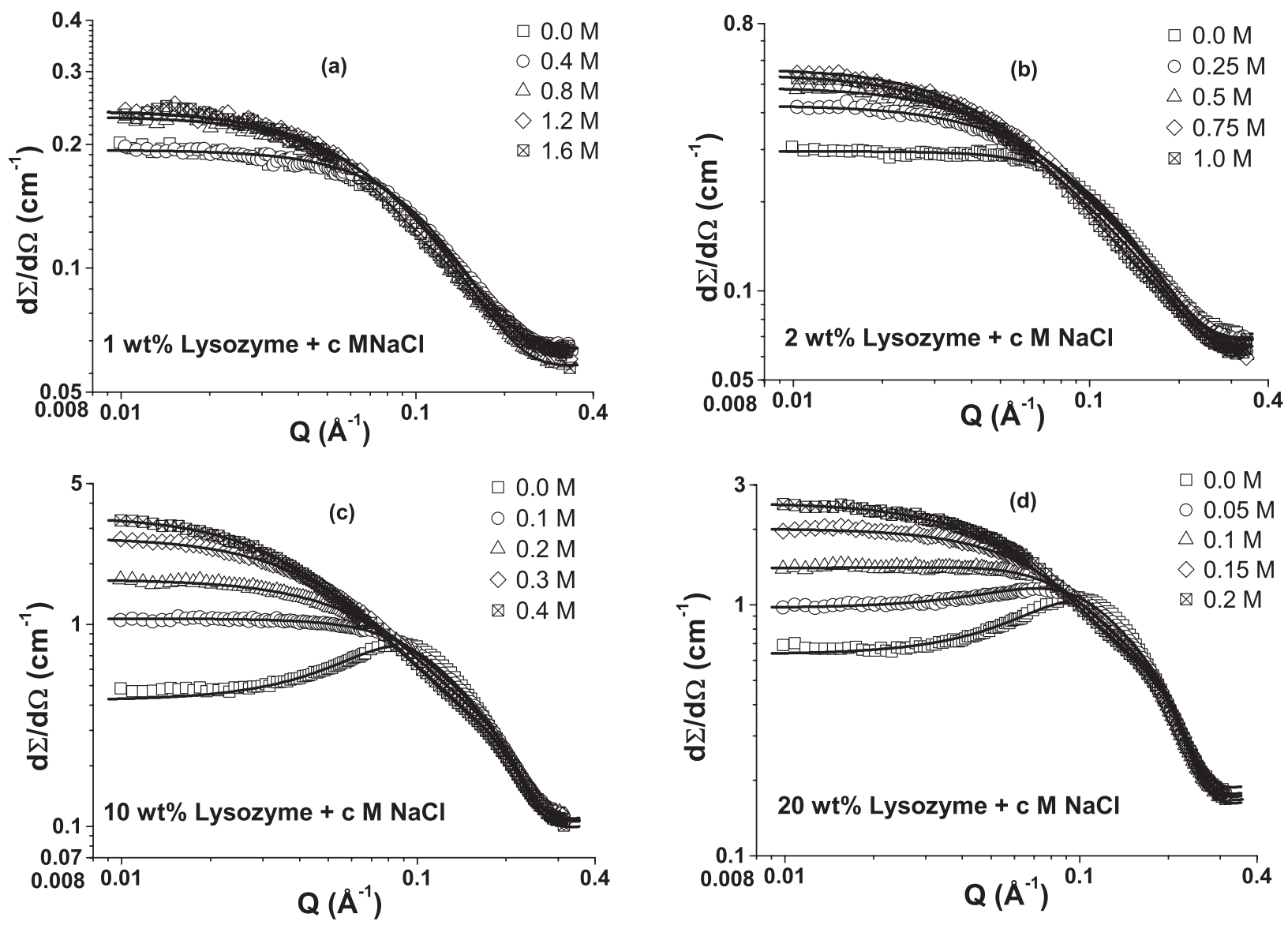

FIG. 4. SANS data of $1,2,10$, and 20 wt $\%$ lysozyme with varying $\mathrm{NaCl}$ concentration. 
LLPT are found to agree reasonably well with the theoretical calculation using Rescaled Mean Spherical Approximation (RMSA) as proposed by Hansen and Hayter [47]. The strength and range of attractive potential related to $K_{1}$ and $Z_{1}$ are found to increase unlike those of repulsion $\left(K_{2}, Z_{2}\right)$ which decrease on addition of salt. These variations suggest that attractive potential tends to be stronger and broader whereas the repulsion becomes weak and narrower with increase in salt concentration. The evolution in the attractive interaction with salt can be understood in terms of enhanced hydrophobic attraction among the protein molecules due to entropy driven dehydration induced by the hydrated salt ions at the protein surface $[48,49]$. On the other hand, the increase in counterion condensation and the ionic strength (screening) are known to decrease the strength and range of the repulsive potential, respectively $[50,51]$. The value of the potential at a distance corresponding to the size of the water molecule [52,53] through which the attractive interaction is believed to be mediating increases with the salt concentration (Table I). This corresponds to a contact distance of about $35 \AA$ between protein molecules, which is the sum of the protein diameter and the size of the water molecule. The LLPT is found to occur when the interaction potential is around $-3.5 \mathrm{kT}$. The interaction potential of this order which is significantly larger than the average kinetic energy $(1.5 \mathrm{kT})$ of the protein macromolecule has also been reported in the literature [29] and could be required for the cases when the interparticle distance is much larger than the size of the particle. This suggests that the protein concentration can be one of the important parameters for tuning interaction to achieve LLPT and is discussed below.

TABLE II. The fitting parameters of $2 Y$ potential for SANS data of $1,2,10$, and $20 \mathrm{wt} \%$ lysozyme. The second virial coefficient $\left(B_{2}\right)$ is calculated from the fitted parameters of the potentials.

\begin{tabular}{|c|c|c|c|c|c|c|}
\hline $\begin{array}{l}\text { Lysozyme } \\
\text { cocentration } \\
(\text { wt } \%)\end{array}$ & $\begin{array}{c}\mathrm{NaCl} \\
\text { concentration } \\
(M)\end{array}$ & $K_{1}$ & $Z_{1}$ & $K_{2}$ & $Z_{2}$ & $\begin{array}{c}B_{2} \\
\left(\mathrm{~mol} \mathrm{ml} / \mathrm{g}^{2}\right)\end{array}$ \\
\hline \multirow[t]{5}{*}{1} & 0.0 & 4.0 & 11.0 & 1.4 & 3.0 & $1.2 \times 10^{-4}$ \\
\hline & 0.4 & 4.5 & 11.0 & 1.4 & 3.5 & $0.4 \times 10^{-4}$ \\
\hline & 0.8 & 7.0 & 6.5 & 0.3 & 9.0 & $-6.5 \times 10^{-4}$ \\
\hline & 1.2 & 7.7 & 6.0 & 0.2 & 10.5 & $-7.9 \times 10^{-4}$ \\
\hline & 1.6 & 8.2 & 6.0 & 0.1 & 12.0 & $-8.5 \times 10^{-4}$ \\
\hline \multirow[t]{5}{*}{2} & 0.0 & 4.0 & 11.0 & 1.5 & 3.0 & $1.5 \times 10^{-4}$ \\
\hline & 0.25 & 6.3 & 8.0 & 0.5 & 6.5 & $-4.3 \times 10^{-4}$ \\
\hline & 0.5 & 7.5 & 7.0 & 0.3 & 7.8 & $-6.4 \times 10^{-4}$ \\
\hline & 0.75 & 8.0 & 6.0 & 0.2 & 8.0 & $-8.2 \times 10^{-4}$ \\
\hline & 1.0 & 8.0 & 6.0 & 0.1 & 8.0 & $-8.3 \times 10^{-4}$ \\
\hline \multirow[t]{5}{*}{10} & 0.0 & 4.0 & 11.0 & 2.3 & 2.2 & $6.1 \times 10^{-4}$ \\
\hline & 0.1 & 4.2 & 11.0 & 1.0 & 4.0 & $-0.6 \times 10^{-4}$ \\
\hline & 0.2 & 4.8 & 11.0 & 0.5 & 5.7 & $-2.0 \times 10^{-4}$ \\
\hline & 0.3 & 5.9 & 10.0 & 0.4 & 6.8 & $-3.1 \times 10^{-4}$ \\
\hline & 0.4 & 6.3 & 9.5 & 0.3 & 7.4 & $-3.7 \times 10^{-4}$ \\
\hline \multirow[t]{5}{*}{20} & 0.0 & 4.0 & 11.0 & 2.3 & 2.1 & $2.7 \times 10^{-4}$ \\
\hline & 0.05 & 4.2 & 11.0 & 1.2 & 3.1 & $0.5 \times 10^{-4}$ \\
\hline & 0.1 & 4.4 & 10.0 & 0.9 & 3.5 & $-0.8 \times 10^{-4}$ \\
\hline & 0.15 & 4.6 & 10.5 & 0.7 & 4.3 & $-1.5 \times 10^{-4}$ \\
\hline & 0.2 & 5.3 & 11.0 & 0.5 & 5.3 & $-2.2 \times 10^{-4}$ \\
\hline
\end{tabular}

The second virial coefficient $\left(B_{2}\right)$ is widely used for understanding phase transitions in protein solutions [54-56]. A solution condition that corresponds to a positive value for $B_{2}$ indicates strong repulsive interaction between the protein molecules. Strong attractive interaction between protein molecules, however, gives rise to large negative values for $B_{2}$ [57]. These solution conditions are responsible for leading to phase transitions such as LLPT. The calculated value of $B_{2}$ for a protein solution undergoing LLPT with increasing salt concentration is given in Table I. The $B_{2}$ has a high positive value $\left(5.9 \times 10^{-4} \mathrm{~mol} \mathrm{ml} / \mathrm{g}^{2}\right)$ for a salt-free protein solution whereas

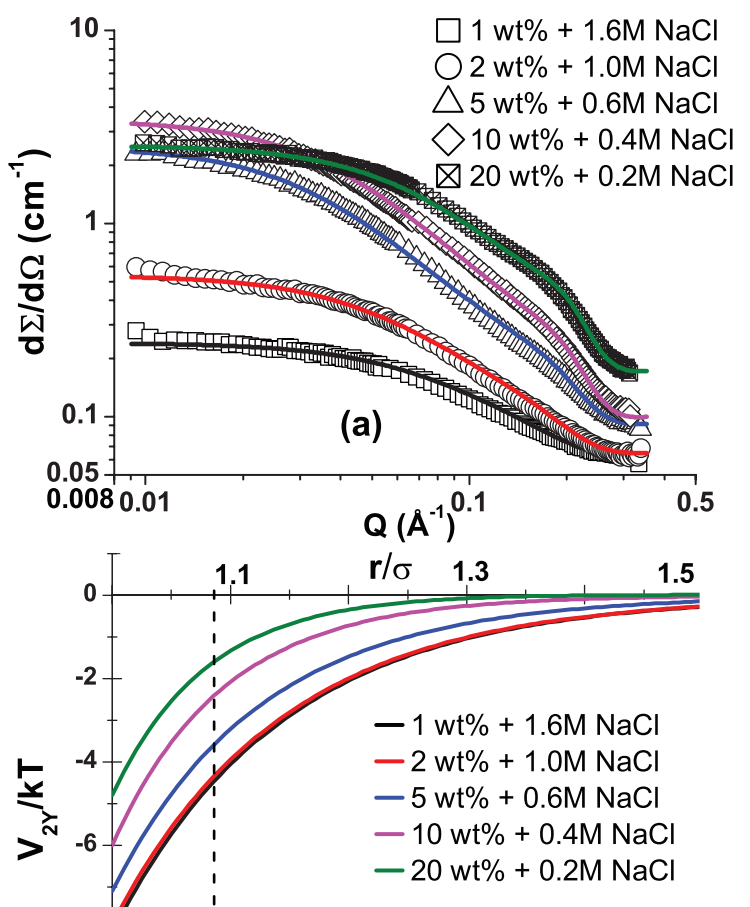

(b)

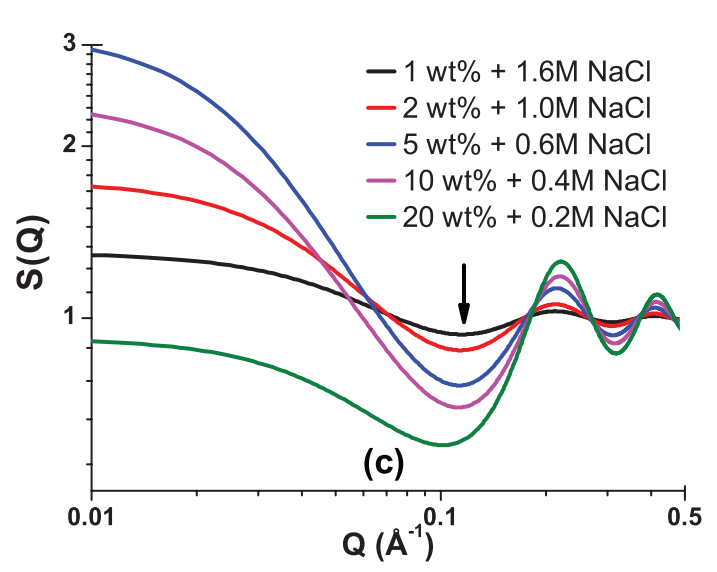

FIG. 5. (Color online) The correlation of (a) SANS data, (b) total potential, and (c) structure factor for different protein concentrations in the presence of $\mathrm{NaCl}$ on approaching LLPT. The curves for total potential bottom to top correspond to increasing protein concentration. In the calculation of structure factor the first minimum as marked from top to bottom corresponds to increasing protein concentration. 
it becomes negative with the addition of salt. The $B_{2}$ value at the LLPT is found to be around $-6.3 \times 10^{-4} \mathrm{~mol} \mathrm{ml} / \mathrm{g}^{2}$, which is consistent with that reported in the literature [15].

Figure 4 shows the SANS data for different protein concentrations $(1,2,10$, and $20 \mathrm{wt} \%)$ with varying concentration of $\mathrm{NaCl}$ approaching their respective LLPT points. The LLPT points are obtained at $\mathrm{NaCl}$ concentrations of $1.65,1.05,0.42$, and $0.22 M$ for $1,2,10$, and 20 wt $\%$ lysozyme solutions, respectively. The correlation peak of these SANS data as observed clearly in the absence of salt shifts to a higher $Q$ value with increasing protein concentration. This is as a result of an increase in number density of macromolecules leading to a decrease in the average distance between the macromolecules with concentration. All the SANS data irrespective of protein concentration approaching LLPT show similar trends to that as discussed in the case of Fig. 2. There is a buildup of scattering in the low $Q$ region with increasing salt concentration. The fitted interaction parameters in these systems are given in Table II. In all the cases, the parameters $K_{1}$ (strength) and $1 / Z_{1}$ (range) of attractive potential increase whereas the parameters $K_{2}$ and $1 / Z_{2}$ of repulsive potential decrease on approaching LLPT. However, the variation of parameters (strength and range) and $B_{2}$ values corresponding to attractive potential is found significantly higher for lower protein concentrations. This may be the deciding factor to correlate the concentration of protein and salt to achieve LLPT, for example, as observed in Fig. 1.

The comparison of SANS data on approaching (prior to) LLPT for different protein concentrations is given in Fig. 5(a). The data are compared for $1 \mathrm{wt} \%$ lysozyme with $1.6 \mathrm{M} \mathrm{NaCl}$ $($ LLPT salt concentration $=1.65 M), 2$ wt \% lysozyme with $1.0 M \mathrm{NaCl}$ (LLPT salt concentration $=1.05 M$ ), $5 \mathrm{wt} \%$ lysozyme with $0.6 M \mathrm{NaCl}$ (LLPT salt concentration = $0.62 M$ ), 10 wt \% lysozyme with $0.4 M \mathrm{NaCl}$ (LLPT salt concentration $=0.42 \mathrm{M}$ ), and $20 \mathrm{wt} \%$ lysozyme with $0.2 \mathrm{M}$ $\mathrm{NaCl}$ (LLPT salt concentration $=0.22 M$ ). It is observed that the data are significantly different depending on the protein concentration. The difference in the data may arise because of a change in the interaction potential and hence the structure factor of the system. The protein concentration dependent fitted interaction potential and the structure factor approaching LLPT are plotted in Figs. 5(b) and 5(c), respectively. The values of potential (at a distance of the size of the water molecule) responsible for LLPT in these systems are given in Table III. The required attractive potential for obtaining LLPT is found to decrease with increasing protein concentration and hence can be achieved at a much lower amount of salt for higher protein concentrations. The lower values of attractive
TABLE III. The protein concentration dependence on the depth of total potential on approaching LLPT. The value of total potential is calculated at a distance that protein molecules experience through a hydration layer between them. The second virial coefficient $\left(B_{2}\right)$ is calculated from the fitted parameters of the potentials.

\begin{tabular}{lccc}
\hline \hline $\begin{array}{l}\text { Protein } \\
\text { concentration } \\
(\mathrm{wt} \%)\end{array}$ & $\begin{array}{c}\text { Salt } \\
\text { concentration } \\
(M)\end{array}$ & $V_{2 \mathrm{Y}} / k T$ & $\begin{array}{c}B_{2} \\
\left(\mathrm{~mol} \mathrm{ml} / \mathrm{g}^{2}\right)\end{array}$ \\
\hline 1 & 1.6 & -4.1 & $-8.5 \times 10^{-4}$ \\
2 & 1.0 & -4.0 & $-8.3 \times 10^{-4}$ \\
5 & 0.6 & -3.5 & $-6.3 \times 10^{-4}$ \\
10 & 0.4 & -2.2 & $-3.7 \times 10^{-4}$ \\
20 & 0.2 & -1.5 & $-2.2 \times 10^{-4}$ \\
\hline \hline
\end{tabular}

potential to achieve LLPT for higher protein concentrations are also consistent with the lower $B_{2}$ values for these systems (Table III). This can be understood on the basis that the average distance between the protein molecules decreases with protein concentration, which makes it easier (lower potential) for molecules to undergo LLPT. The present study thus shows that the average distance between particles is an important parameter and can be simply varied by the particle concentration in tuning the LLPT.

\section{CONCLUSIONS}

SANS has been used to study the structure and interaction in aqueous solution of lysozyme protein leading to liquid-liquid phase transition (LLPT) as induced by salt. The LLPT is found to be governed by the change in the interaction potential of protein molecules whereas the conformational structure of the protein remains unaltered. The interaction is modeled by the two Yukawa potential combining short-range attraction and long-range repulsion. The LLPT occurs as a result of the dominance of a non-DLVO kind of short-range attraction with increasing salt concentration. This attractive interaction is believed to arise from entropy driven dehydration induced by the hydrated salt ions. The magnitude of attractive potential required to achieve LLPT is of the order of average kinetic energy of the molecules but decreases significantly with increasing protein concentration.

\section{ACKNOWLEDGMENTS}

This work is based on the SANS experiments performed at the Swiss Spallation Neutron Source SINQ, Paul Scherrer Institut, Villigen, Switzerland.
[1] J. B. Rouget, T. Aksel, J. Roche, J. L. Saldana, A. E. Garcia, D. Barrick, and C. A. Royer, J. Am. Chem. Soc. 133, 6020 (2011).

[2] J. A. Brannigan, G. Dodson, H. J. Duggleby, P. C. E. Moody, J. L. Smith, D. R. Tomchick, and A. G. Murzin, Nature 378, 416 (1995).

[3] K. A. Dawson, Curr. Opin. Colloid Interface Sci. 7, 218 (2002).

[4] N. Asherie, Methods 34, 266 (2004).
[5] J. N. Israelachvili, Intermolecular and Surface Forces (Academic Press, New York, 1992).

[6] R. P. Sear, Curr. Opin. Colloid Interface Sci. 11, 35 (2006).

[7] A. Stradner, H. Sedgwick, F. Cardinaux, W. C. K. Poon, S. U. Egelhaaf, and P. Schurtenberger, Nature 432, 492 (2004).

[8] Y. Liu, E. Fratini, P. Baglioni, W.-R. Chen, and S.-H. Chen, Phys. Rev. Lett. 95, 118102 (2005). 
[9] A. Tardieu, A. Le Verge, M. Malfois, F. Bonnete, S. Finet, M. Ries-Kautt, and L. Belloni, J. Cryst. Growth 196, 193 (1999).

[10] A. Ducruix, J. P. Guilloteau, M. Ries-Kautt, and A. Tardieu, J. Cryst. Growth 168, 28 (1996).

[11] M. Malfois, F. Bonnete, L. Belloni, and A. Tardieu, J. Chem. Phys. 105, 3290 (1996).

[12] A. C. Dumetz, A. M. Chockla, E. W. Kaler, and A. M. Lenhoff, Biophys. J. 94, 570 (2008).

[13] D. Rosenbaum, P. C. Zamora, and C. F. Zukoski, Phys. Rev. Lett. 76, 150 (1996).

[14] M. Muschol and F. Rosenberger, J. Chem. Phys. 107, 1953 (1997).

[15] F. Bonnete, S. Finet, and A. Tardieu, J. Cryst. Growth 196, 403 (1999).

[16] P. R. ten Wolde and D. Frenkel, Science 277, 1975 (1997).

[17] H. Bloemendal, W. de Jong, R. Jaenicke, N. H. Lubsen, C. Slingsby, and A. Tardieu, Prog. Biophys. Mol. Biol. 86, 407 (2004).

[18] J. A. Thomson, P. Schurtenberger, G. M. Thurston, and G. B. Benedek, Proc. Natl. Acad. Sci. USA 84, 7079 (1987).

[19] Y. Wang and O. Annunziata, Langmuir 24, 2799 (2008).

[20] J. Lu, K. Carpenter, R.-J. Li, X.-J. Wang, and C.-B. Ching, Biophys. Chem. 109, 105 (2004).

[21] A. Shukla, E. Mylonas, E. Di Cola, S. Finet, P. Timmins, T. Narayanan, and D. I. Svergun, Proc. Natl. Acad. Sci. USA 105, 5075 (2008).

[22] S. Chodankar and V. K. Aswal, Phys. Rev. E 72, 041931 (2005).

[23] Y. Minezaki, N. Niimura, M. Ataka, and T. Katsura, Biophys. Chem. 58, 355 (1996).

[24] G. Pellicane, D. Costa, and C. Caccamo, J. Phys.: Condens. Matter 15, 375 (2003).

[25] D. N. Petsev and P. G. Vekilov, Phys. Rev. Lett. 84, 1339 (2000).

[26] J. N. Israelachvili and H. Wennerstrom, Nature 379, 219 (1996).

[27] S. Finet, F. Skouri-Panet, M. Casselyn, F. Bonnete, and A. Tardieu, Curr. Opin. Colloid Interface Sci. 9, 112 (2004).

[28] M. Boström, D. R. M. Williams, and B. W. Ninham, Phys. Rev. Lett. 87, 168103 (2001).

[29] F. Zhang, R. Roth, M. Wolf, F. Roosen-Runge, M. W. A. Skoda, R. M. J. Jacobs, M. Stzuckie, and F. Schreiber, Soft Matter 8, 1313 (2012).

[30] R. J. Baxter, J. Chem. Phys. 49, 2770 (1968).

[31] S. V. G. Menon, C. Manohar, and K. Srinivasa Rao, J. Chem. Phys. 95, 9186 (1991).
[32] Y. Liu, W.-R. Chen, and S.-H. Chen, J. Chem. Phys. 122, 044507 (2005).

[33] M. L. Broide, T. M. Tomic, and M. D. Saxowsky, Phys. Rev. E 53, 6325 (1996).

[34] S.-H. Chen, Annu. Rev. Phys. Chem. 37, 351 (1986).

[35] K. Mortensen, Curr. Opin. Colloid Interface Sci. 3, 12 (1998).

[36] J. Kohlbrecher and W. Wagner, J. Appl. Crystallogr. 33, 804 (2000).

[37] U. Keiderling, Appl. Phys. A 74, s1455 (2002).

[38] W. G. McMillan and J. E. Mayer, J. Chem. Phys. 13, 276 (1945).

[39] P. R. Bevington, Data Reduction and Error Analysis for Physical Sciences (McGraw-Hill, New York, 1969).

[40] A. P. Golovanov, G. M. Hautbergue, S. A. Wilson, and L.-Y. Lian, J. Am. Chem. Soc. 126, 8933 (2004).

[41] V. K. Aswal and J. Kohlbrecher, Chem. Phys. Lett. 425, 118 (2006).

[42] A. J. Chinchalikar, V. K. Aswal, J. Kohlbrecher, and A. G. Wagh, Eur. Phys. J. E: Soft Matter Biol. Phys. 35, 55 (2012).

[43] S. Kumar, V. K. Aswal, and J. Kohlbrecher, Langmuir 27, 10167 (2011).

[44] N. Kovalchuk, V. Starov, P. Langston, and N. Hilal, Adv. Colloid Interface Sci. 147-148, 144 (2009).

[45] S. Beretta, G. Chirico, and G. Baldini, Macromolecules 33, 8663 (2000).

[46] Y. Liu, L. Porcar, J. Chen, W.-R. Chen, P. Falus, A. Faraone, E. Fratini, K. Hong, and P. Baglioni, J. Phys. Chem. B 115, 7238 (2011).

[47] J. P. Hansen and J. B. Hayter, Mol. Phys. 46, 651 (1982).

[48] E. E. Meyer, K. J. Rosenberg, and J. N. Israelachvili, Proc. Natl. Acad. Sci. USA 103, 15739 (2006).

[49] C. J. V. Oss, J. Mol. Recognit. 16, 177 (2003).

[50] V. K. Aswal and P. S. Goyal, Chem. Phys. Lett. 368, 59 (2003).

[51] V. K. Aswal and P. S. Goyal, Phys. Rev. E 67, 051401 (2003).

[52] P. S. Goyal, S. V. G. Menon, B. A. Dasannacharya, and P. Thiyagarajan, Phys. Rev. E 51, 2308 (1995).

[53] A. J. Chinchalikar, V. K. Aswal, J. Kohlbrecher, and A. G. Wagh, Colloid Polym. Sci. 288, 1601 (2010).

[54] A. George and W. W. Wilson, Acta Crystallogr. D 50, 361 (1994).

[55] H. Elcock and J. A. McCammon, Biophys. J. 80, 613 (2001).

[56] B. L. Neal, D. Asthagiri, O. D. Velev, A. M. Lenhoff, and E. W. Kaler, J. Cryst. Growth 196, 377 (1999).

[57] B. Guo, S. Kao, H. McDonald, A. Asanov, L. L. Combs, and W. W. Wilson, J. Cryst. Growth 196, 424 (1999). 